de l'axe des abcisses, à travers lesquelles passent des courroies munies de boucles se fixant, par dessous, au moyen de boucles de laiton et de ressorts. De la sorte la Carpe est maintenue solidement et on peut tout à son aise vaquer aux mensurations (Fig. $7^{8}$ ).

Pour la détermination de la largeur du corps on utilise un compas d'épaisseur ordinaire à une seule branche longue d'une dizaine de centimètres. Le pied de cet appareil, élargi pour lui donner de l'assiette et dont la base forme l'origine de la graduation de la tige, se pose sur la table ; on abaisse ensuite la branche mobile; quand elle appuie sur le flanc du poisson, on la fixe au moyen d'une vis de pression et on fait la lecture.

Ces opérations effectuées, il ne reste plus qu'à procéder à la pesée, pour laquelle on emploie une simple balance de cuisine. Outre le poids total, Krizenecky a déterminé séparément celui des viscères, des glandes sexuelles, de la tête, des nageoires, des écailles, de la peau et du squelette, mais les constatations faites sur l'importance relative des divers organes n'ont pas encore été publiées.

(A suivre).

\title{
LES PRINCIPES DE LA LIMNOLOGIE
}

\author{
Par M. Paul VIVIER \\ Garde général des Eaux et Forêts, \\ Assistant à la Station de recherches de l'Ecole Nationale des Eaux et Forêts.
}

(Suite) (1)

III. - Les différentes régions d'un lac; leur colonisation vivante (suite).

De nombreuses expériences de laboratoire ont été faites sur l'action de ces deux influences physiques si intéressantes, le thermo et le photo-tropisme; il serait trop long de les rapporter ici (2).

Parmi les circonstances chimiques qui agissent sur le plancton, il faut citer les sels de fer dont l'influence est nette sur le développement des bactéries; l'action de l'oxygène est plus vive sur le poisson que sur les Entomostracés qui se contentent en génę́ral d'une tencur supérieure seulement à 0,2 cc. par litre; dans des eaux sans oxygène, à hydrogène sulfuré, vivent encore certains Infusoires et des larves de Corethra; l'action du gaz carbonique est, on le sait, considérable sur les végćtaux : la diminution

(1) Voir Bulletin :- $n^{\circ} 68$, Février 1934, p. I1 $;-n^{\circ} 69$, Mars 1934, p. 245.

(2) Rose (M.). - La question des tropismes. - les Presses universitaires de France, Paris.

SIEDEntop (W.). - Physiologische Beobachtungen an Leptodora Kindtii. 
des Diatomées, parfois observée au début de l'été, paraît due à l'épuisement de ce gaz dans les couches d'eau supérieures; leur réapparition, au

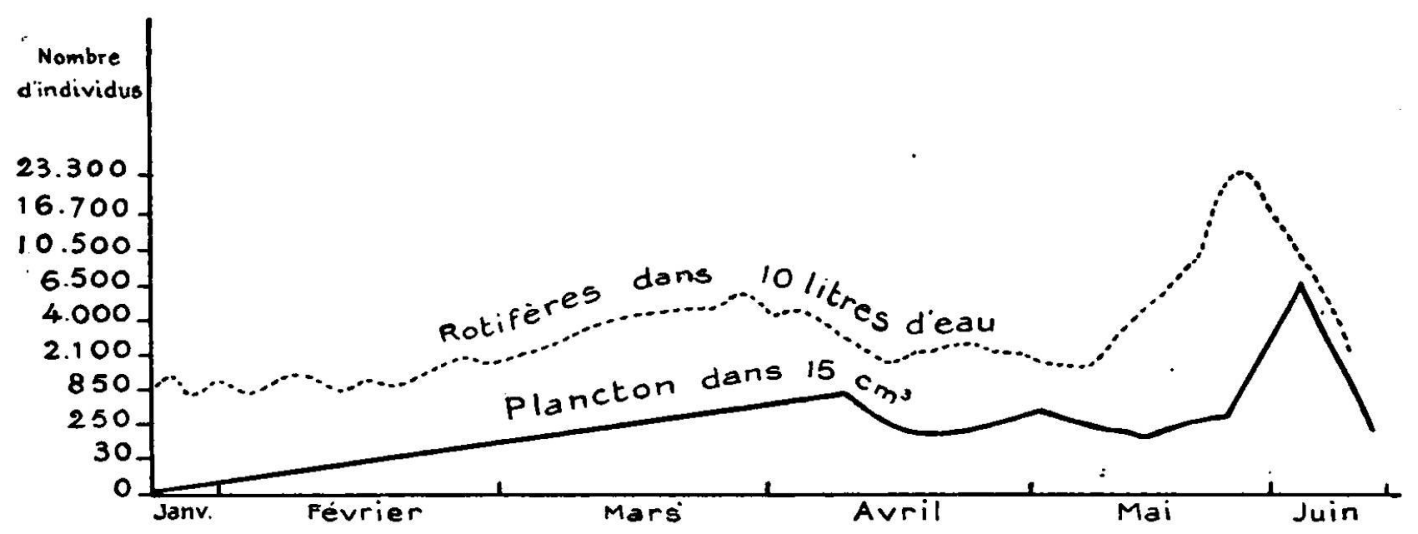

Fig. 79. - Rapport entre le plancton (nannoplancton) et les Rotifères dans un étang (Holzteich).

(D'après Diffenbacii).

début de l'hiver, serait liée aux courants thermiques qui ramènent le gaz carbonique des couches profondes où il s'est formé par décomposition du calcaire des Diatomées estivales.

Enfin le phytoplancton qui sert de nourriture aux animaux du zooplancton et prinoipalement aux Rotifères, commande les variations de ceux-ci (fig. 79).

Les variations annuelles du plancton dépendent donc de tous ces facteurs physiques, chimiques et biologiques; ainsi dans le grand lac de Plön, on a distingué de Décembre à Juillet une période de Bactériacées à laquelle succède, de Juillet à Novembre, une ère à Schizophycées ; de même, parmi les Entomostracés certaines es-

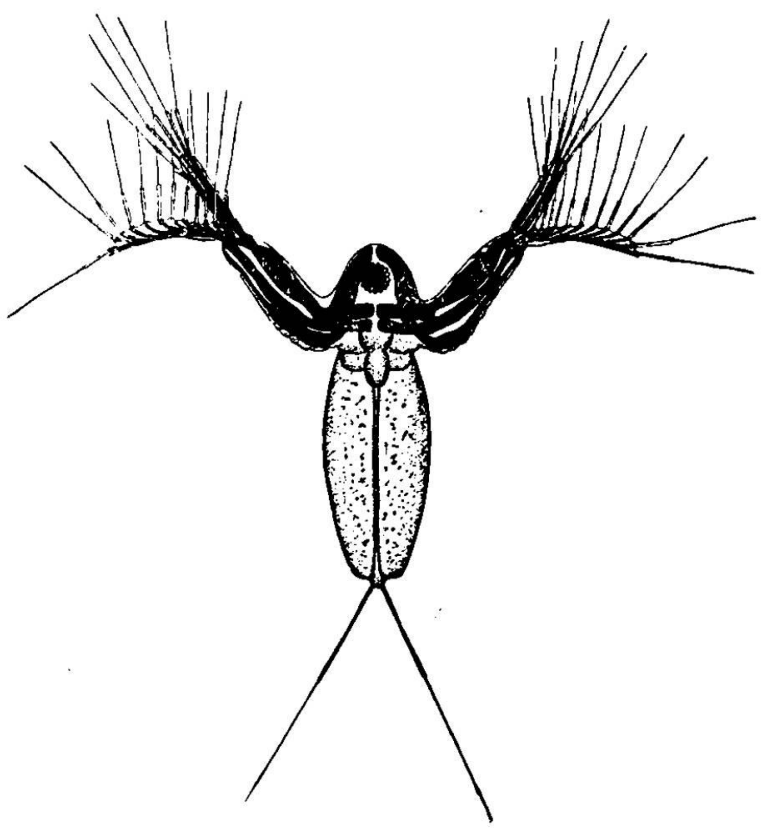

Fig. 80. - Diaphanosoma brachyurum.

Espèce caractéristique du plancton, surtout en été.

(D'après C. Wesenberg-Lund). pèces paraissent prédominantes certains mois : ainsi Diaptomus, en MarsAvril, Bosmina, en Janvier, Juin et Juillet, Daphnia, en Aout et Septembre, Cyclops, en Mai, Octobre, Novembre et Décembre (HAEmpel). 
Certaines espèces du plancton, on l'a vu plus haut, sont littorales : Sida crystallina (O.-F. MüLler), Scapholeberis mucronata (O.-F. MüLLER), etc. ; d'autres nettement pélagiques : Bithotrephes longimanus (fig. 72) (F. LEYDIG), Leptodora Kindtii (fig. 7 I) (FockE), la plupart sont saisonnières : Diaphanosoma (fig. 8o), Daphnia (fig. 81), Leptodora, Bithotrephes, Folyarthra et la plupart des Rotifères ; quelques-unes pérennes : Diapto-

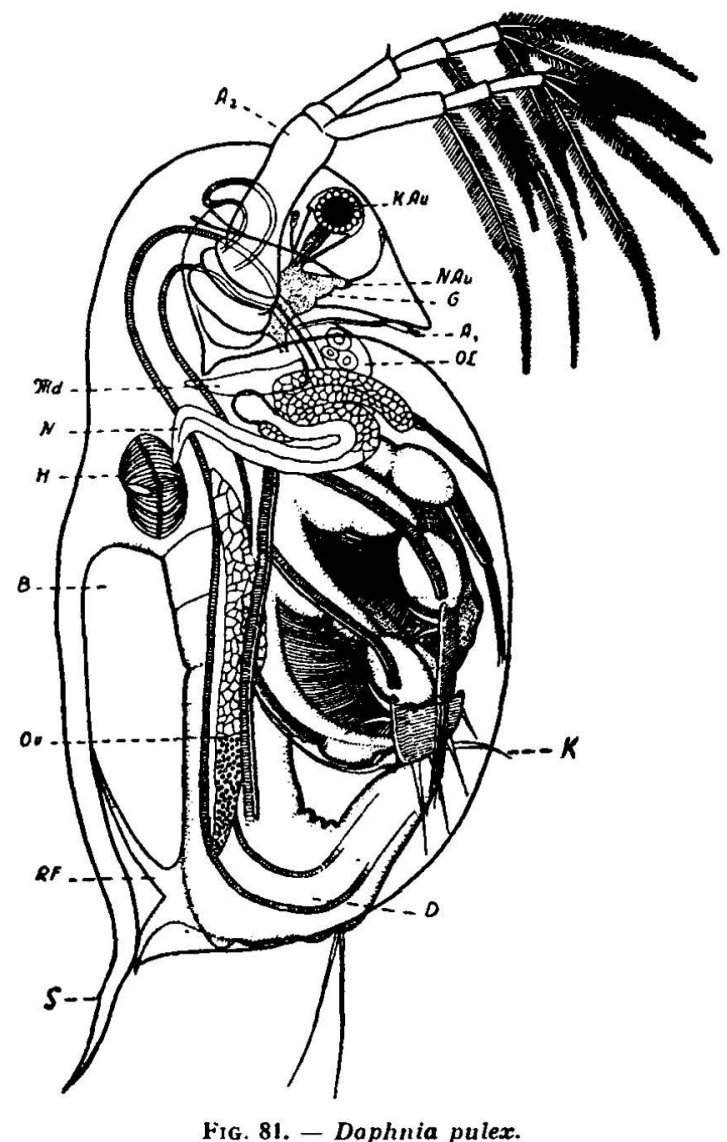

A1 : première paire dantennes, organe sensitif; - A2 : seconde paire d'antennes, organe propulseur; - $B$ poche incubatrice; - $\mathbf{D}$ : intestin ; - $\mathbf{G}$ : cerveau; $-\mathrm{H}$ : cour ; - K : griffe cauda.e; - K Au oil composé ; $\mathbf{N}$ : glande; - N Au : œí, frontal ; - Md : mandibule ;

OL: labre; - OV : ovaire; - RF : protuberance iraversant ia poche incub.ttrice; $-\mathrm{S}$ : aiguillon. mus, Cyclops, Notolca, Anurea; d'autres indifférentes : Bosmina Coregoni (BAIRD), Heterocope Weismanni (SchmeIL).

La répartition du zooplancton en profondeur est la suivante, dans les lacs subalpins :

de 0 à 2 mètres : grande pauvreté ;

de 2 à $3 o$ mètres (été) et à jo mètres (hiver) : richesse ; au-dessous de 50 mètres : très grande pauvreté.

Dans les lacs baltiques et humiques, le zooplancton, au contraire, paraît être nettement abondant en surface.

Dans le lac des QuatreCantons, Burckhardt distingue quatre zones :

Une zone à Rotifères de 5 à Io mètres.

Une zone à Cladocères de Io à 30 mètres.

Une zone à Copépodes de 3o à 70 mètres.

Une zone abyssale, à peu prèsıdépourvue de plancton, de 80 à 100 mètres.

Les animaux qui vivent le plus profondément sont les Copépodes et particulièrement le genre Cyclops. Cyclops viridis (JuRINE) est rencontré communément dans les estomacs de lottes pêchées dans les fonds voisins de 200 mètres (lac Léman) (Vivier).

Le phytoplancton, par suite de l'absence totale de lumière, est inexistant Jans les grands fonds ; en été cependant on trouve les Diatomées jusqu'à iz mètres et en hiver jusqu'à 80 mètres; les Characées se rencontrent à 
25 mètres (ForzL) et la mousse Hypnum Lemani vit à 6o mètres de fond (lac Léman).

On a fait d'assez nombreuses évaluations de quantités d'individus, d'espèces ou de groupes planctoniques contenus dans un volume connu d'eau. Les résultats, on le conçoit, varient beaucoup avec les lacs ; THIEnEmanN donne une moyenne de r.ooo individus par litre d'eau dans les lacs de l'Eifel et un maximum de roo.00o sous la glace dans les lacs baltiques ; dans un lac très riche comme le Dobersdorfersee, on trouve $198 \mathrm{~cm}^{3}$ de plancton par mètre cube d'eau tandis que dans le lac de Neuchâtel il n'y aurait que $5 \mathrm{~cm}^{3}$. Les Algues ont parfois un développement extraordinaire. (2.80o kilos (poids sec) dans un petit lac allemand, le Lietzensee).

Le rôle du plancton est considérable : non seulement les Algues vertes fixent le gaz carbonique pour dégager de l'oxygène si utile à la vie, mais le phytoplancton en général sert de nourriture aux animaux et particulièrement aux Rotifêres, qui à leur tour sont mangés par les Crustacés ou par les Poissons. On a fait de nombreuses études sur l'alimentation des Poissons ; chez la plupart on retrouve les éléments du plancton, particulièrement chez les Corégones qui font des Entomostracés leur nourriture presque exclusive ; l'Omble-Chevalier mange aussi des Mollusques (Pisidium) et des larves de Chironomides ; la Lotte du Léman affectionne le Cyclops Viridis (Junine), le jeune Gardon (Leuciscus rutilus L.) dans le lac Nantua mange beaucoup de Rotifères et de Péridiniens (LÉGRR) ; le ChevaineMeunier aime particulièrement les Entomostracés, mais prend aussi des Diatomées et des Algues ; la Perche est omniphage (Funrmanv).

Lorsqu'on ouvre un estomac de Poisson, on constate en général la présence de nombreux individus d'une même espèce. Cette remarque a été faite depuis longtemps pour les Corégones bourrés de Leptodora et de Bithotrephes, toujours rares dans les filets à plancton; on trouve de même chez l'Omble et chez la Perche de très nombreux spécimens de Sida crystallina (O. F. M.) par exemple (Vivier). On en avait déduit que les Poissons et surtout les Corégones choisissaient leur nourriture, dans le plus menu détail ; il est plus probable d'admettre que les espèces planctoniques d'Entomostracés vivent en essaims, que les filets de capture n'ont pas permis de déceler d'une façon précise. Cette question, en tout cas, n'est pas complètement résolue et il serait téméraire d'être affirmatif.

\section{IV. - Classification biologique des Lacs.}

En comparant les caractères chimiques, physiques et biologiques des lacs, Naumann et Thienemann sont arrivés à les répartir en une classification très intéressante :

On appelle lacs eutrophes, des lacs relativement peu profonds, aux bords plats et recouverts d'une large ceinture de végétation aquatique, aux fonds couverts d'une vase riche en matières organiques et facilement putrescible ; l'extrême pauvreté des fonds en oxygène, la présence constante des larves du genre Chironomus, générale des larves de Corethra, la teinte des 
eaux variant du vert au brun-vert, leur alcalinité, leur grande richesse en plancton, caractérisent très bien ce premier type de lac, représenté chez nous par les lacs des Vosges et de Nantua (fig. 82).

On appelle, au contraire, lacs oligotrophes, des lacs profonds, à beine étroite, sans grande végétation aquatique, aux eaux claires et bleues, relativement pauvres en phosphore, en azote et en plancton ; la richesse des fonds en oxygène, la présence constante de l'association du Tanytarsus, l'absence de Corethra, la pauvreté de la faune profonde, tels sont les principaux caractères de ce type de lacs, représenté chez nous par les lacs subalpins : Léman, lac d'Annecy, lac du Bourget (fig. 83).

On appelle enfin lacs dystrophes, des lacs riches en humus, de teinte brune, aux fonds bourbeux et sans oxygène, qui nourrissent l'association

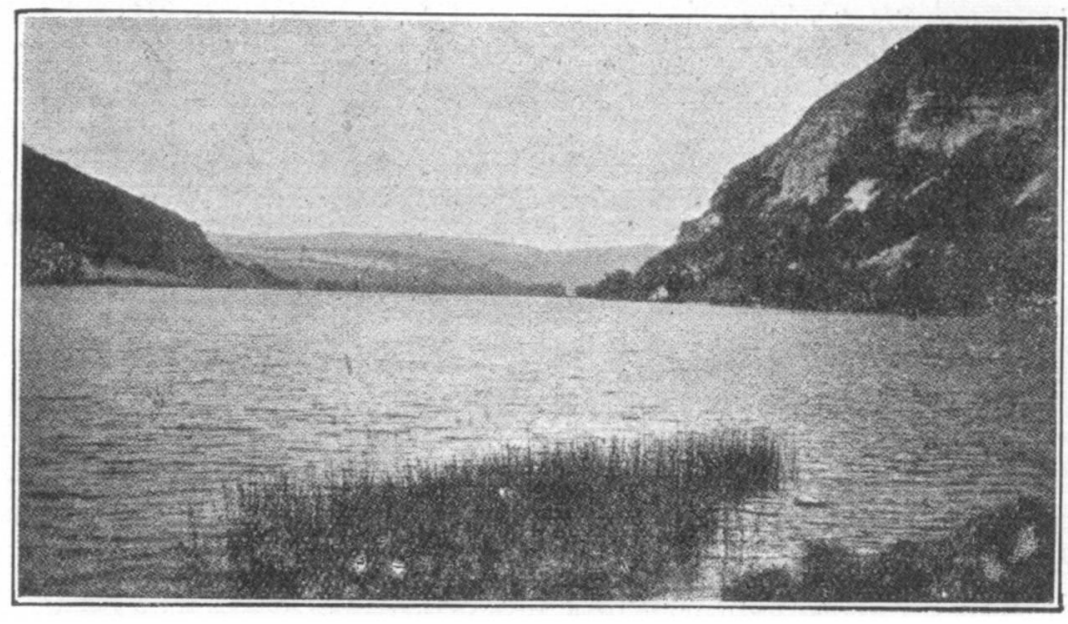

Fig. 82 . - Type de lac eutrophe : le lac de Nantua.
On notera le développement assez considérable de la végétation littorale (Scirpaie).

Corethra-Chironomus plumosus; ce type de lac est très voisin de nos étangs de plaine (Lac de Lispach) (fig. 84).

D'une façon générale, les lacs oligotrophes conviennent aux Salmonidés ; les lacs eutrophes aux peuplements mixtes et aux Cyprinidés.

On a distingué encore dans les lacs oligotrophes (IIAEmprL) : - les lacs sténo-oligotrophés répartis en lacs à Truites de ruisseau, lacs à Omble-Chevalier, lacs à Corégones; - Ies lacs mésoligolrophes dont le peuplement romprend, avec les Cyprinidés, le Sandre et la Truite de lac ; - lacs oligotrophes-type au peuplement mixle (Perche, Brochet, Cyprinidés) où les Corégones sont l'exception.

$$
\text { V. - L'Etang ; sa colonisation. }
$$

Nous avons vu que la caractéristique de l'étang était sa colonisation totale par la flore littorale des lacs ; la lumière pénètre partout ; la végétation est donc abondante. La température, sous notre climat, est froide 
l'hiver, chaude l'été : d'où une plus grande abondance d'animaux en cette saison ; les courants n'existent pas ; la richesse en oxygène est varia-

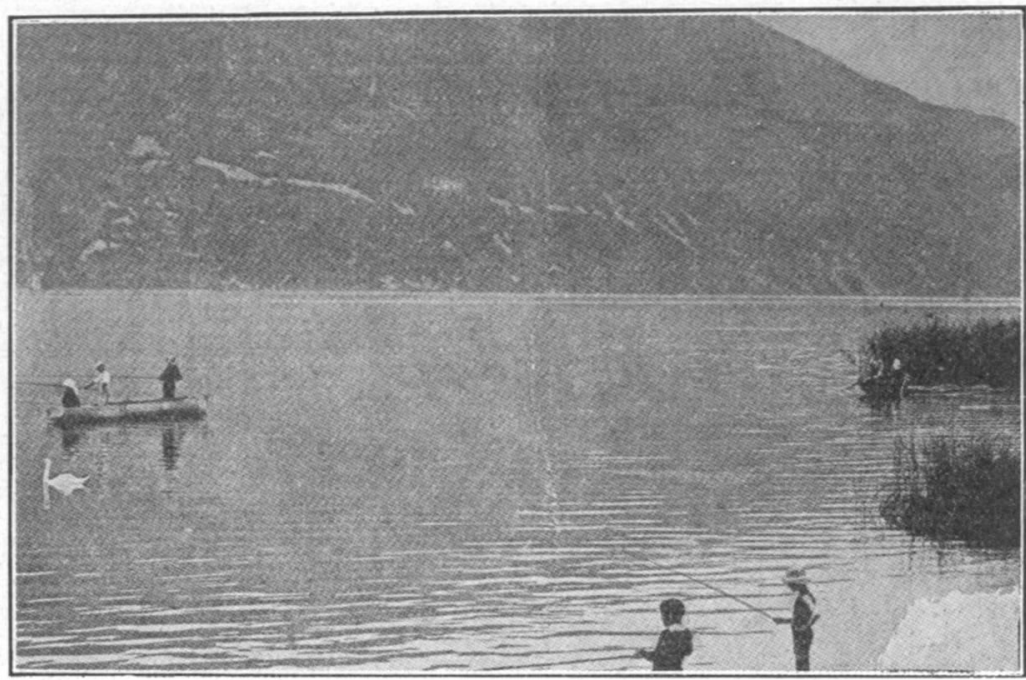

Fig. 83. - Type de lac oligotrophe : le lac du Bourget. Grande profondeur; végétation littorale peu abondante.

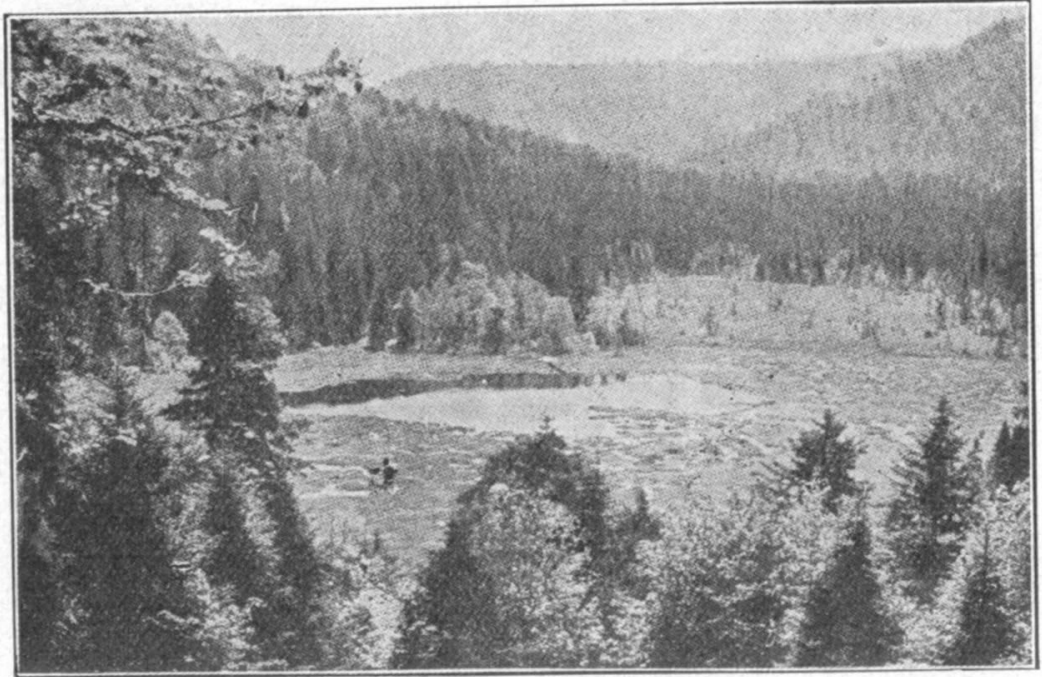

Photo RoL.

Fig. 84. - Type de lac dystrophe : le lac de Lispach dans les Vosges. Le lac est presque entièrement envahi par des îlots de sphaignes.

ble ; la faune se rapproche de celle que nous avons schématisée dans la description d'un littoral lacustre tranquille. Les étangs de France les plus importants, Dombes et Sologne, sont encore assez mal connus dans leur ensemble. 
Pelosse n'a étudié, dans les étangs de la Dombes, que les Entomostracés : il a rencontré une faune riche en espèces et en individus (neuf espèces de Cyclops, Daphnia longispina (fig. 85) et Daphnia pulex, les genres Pleuroxus, Rhynchotalona, Simocephalus, Diaphanosoma, Acroperus, Moina, Canthocamptus). L'hiver il y aurait prédominance de

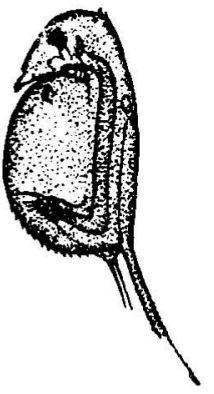

Fig. 85. - Mâle de Daphnia longispina du lac des Quatre-Cantons (Suisse). - Dans la nature, cette espece se reproduit par parthénogénèse $\mathrm{et}$, normalement, re noode de multiplication donn uniquement des femelles. Dans certaines conditions d'expérimentation, on arrive à provoque lapparition des malles. - Grossissement $\times 32$. Daphnia longispina, espèce lacustre, l'été de Bosmina longirostris (O.-F. MULLER).

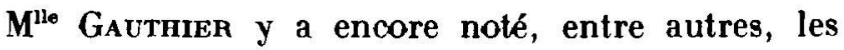
genres suivants :

Algues : Anabøna, Aphanizomenon, Sphœrocystis, Staurastrum, Closterium, Spirogyra.

Diatomés : Cymbella, Synedra, Gonphonema, $\mathrm{Na-}$ vicula, Fragillaria.

Flagellés : Volvox.

Péridiniens : Ceratium.

Rotifères : Brachionus, Polyarthra, Rattulus, Anurea, Asplanchna.

Larves diverses d'insectes, statoblastes de pluma. telles et de cristatelles.

Roy, dans les étangs des environs de la Flèche, a retrouvé les espèces communes d'Entomostracés, et encore quelques autres. Peracantha truncata (0.-

F. M.), Elaphoidella bidens, Dunvehedia crassa (KING), Iliocryptus sordidus (LiÉvis), Lathonura rectirostris (O.-F. M.), Streblocerus serricaudatus (Frscher) ; Cyclops strenuus (Frscher), sténotherme lié au froid, est cons. tant l'hiver ; Cyclops leuckarti (Crads), commun l'été.

Dans les étangs vivent aussi de nombreuses Bactéries qui auraient non seulement une action de nitrification à partir de l'azote atmosphérique, mais aussi parfois de dénitrification, comme les Bactéries du sol ; elles seraient donc généralement enrichissantes ; de plus, elles offrent une nourriture recherchée par la plupart des animaux inférieurs : Amibes, Infusoires, Rotifères, Mollusques et Crustacés.

Dans les étangs de plaine, par suite des actions de putréfaction, dans un milieu organique très riche, la teneur en oxygène doit être toujours faible : aussi ceux-ci ne contiennent-ils que les espèces de poissons les moins exigeants, les Cyprinidés ; dans les étangs de moṇtagne au contraire, où l'eau est constamment renouvelée, et toujours à une assez faible température, vivent parfaitement des Truites.

Il est regrettable que des études plus approfondies n'aient pas été faites sur des eaux stagnantes, d'une aussi grande importance piscicole, qui, pourtant, sous le rapport de la productịon, sont parfois si bien ạménagées. 
VI. - Le Lac, unité vivante; application.

Tous les facteurs physiques, chimiques, biologiques que nous avons esquissés plus haut, très sommairement, agissent les uns sur les autres pour perpétuer la vie d'un lac, de même que dans l'être humain toutes les fonctions concourent à la conservation de l'espèce. Le tableau suivant montre schématiquement leurs rapports réciproques (fig. 86).

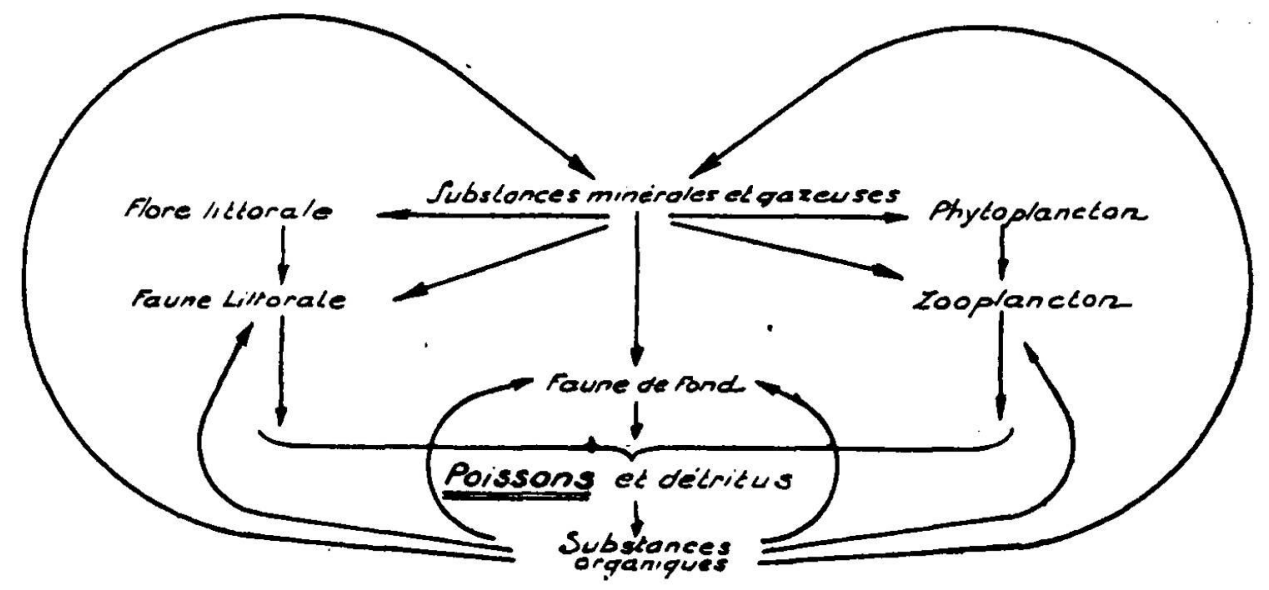

Fia. 86. - Relations entre les divers facteurs qui régissent la vie aquatique dans un lac.

Etant donnée la richesse des sources de vie, minérales et gazeuses, on voit qu'on peut prélever, sur le lot vivant, un fruit, sans appauvrissement notable de l'ensemble; ce fruit, c'est le poisson. Ce revenu, il faut lẹ rechercher le plus constant et aussi le meilleur possible. Kreitmann a montré comment il était possible d'aménager un lac, un peu comme on aménage une forêt ; sans doute la difficulté en est beaucoup plus grande, car il n'est pas possible de recenser exactement un peuplement piscicole. On peut cependant avoir une idée suffisante de l'évolution des espèces et du peuplement général grâce à des statistiques sérieusement effectuées. Il faudra ensuite déterminer la composition d'un peuplement normal qu'on déduira précisément des recherches limnologiques théoriques : température, oxygène, substances dissoutes, variations de niveau, faune, flore, etc. Il sera nécessaire alors de connaître le revenu considéré comme normal, qui, faute de mieux, s'établira par comparaison entre lacs analogues. Le rapprochement des données du peuplement théorique et du revenu idéal de celles du peuplement et du revenu réels du lac considéré, permettra de modifier par la réglementation d'une part et la pisciculture de l'autre l'état existant pour un rendement meilleur. On voit là tout l'intérêt et toute l'importance de ces principes qui ont été appliqués avec fruit au Léman et qui découlent des études limnologiques.

Puissent ces quelques pages d'essai d'initiation à des études nouvelles, en faire comprendre toute la portée pratique.

(A suivre). 